\title{
Aprendizaje procedimental en empresarios Caso Ciudad Juárez, Chihuahua, y Cozumel, Quintana Roo
}

Recibido: 16/09/2016 · Aceptado: 24/01/2017

\author{
Oscar Galván Mendoza* \\ Ricardo Melgoza Ramos \\ Universidad Autónoma de Ciudad Juárez
}

\section{Resumen}

En la literatura dedicada al estudio del emprendimiento se observa una falta de comprensión acerca de cómo un empresario aprende a serlo; por tal motivo, en este trabajo se abordan las experiencias de empresarios desde la perspectiva del aprendizaje procedimental a partir de sus vivencias en el entorno laboral, lo cual se fundamenta con la teoría del desarrollo cognoscitivo de Piaget. La presente es una investigación descriptiva, exploratoria y no experimental, con un enfoque cualitativo, cuyo objetivo fue analizar el aprendizaje procedimental de doce empresarios, de los cuales seis radican en Ciudad Juárez, Chihuahua, y seis en Cozumel, Quintana Roo. Para recabar información se efectuaron entrevistas semiestructuradas en las que se utilizó como guía un instrumento elaborado con base en el trabajo de investigación educativa de Marzano. Para el análisis de la información se empleó la estrategia de estudio de caso múltiple. Finalmente, con los resultados se construyeron cinco dimensiones de aprendizaje procedimental enfocadas a la dinámica empresarial: actitudes y percepciones positivas sobre el aprendizaje; adquisición e integración del conocimiento; refinamiento y profundización del conocimiento, aplicación significativa del conocimiento; y hábitos mentales productivos.

Palabras clave: Ciudad Juárez, Cozumel, aprendizaje procedimental, emprendimiento, empresarios.

*Correo electrónico: noccarempo@gmail.com 


\title{
Procedural learning of businessmen Cases from Ciudad Juárez, Chihuahua and Cozumel, Quintana Roo
}

Recieved: 16/09/2016 · Accepted: 24/01/2017

\author{
Oscar Galván Mendoza* \\ Ricardo Melgoza Ramos \\ Universidad Autónoma de Ciudad Juárez
}

\begin{abstract}
In the literature on the study of entrepreneurship, a lack of comprehension on how the businessman learns to become one can be observed; therefore the experiences of businessmen are explored from the perspective of a procedural learning process based on Piaget's theory of cognitive development. This is a descriptive, exploratory and not experimental research; under a qualitative approach with the objective of analyzing the procedural learning process of twelve businessmen, six from Ciudad Juárez, Chihuahua and six from Cozumel, Quintana Roo. To gather information semi-structured interviews were conducted using Marzano's educational research work as an interview guide. The strategy of multiple case studies was used for the analysis of the information. Based on the results five dimensions of procedural learning process focused on business dynamics were constructed: Positive attitudes and perceptions about learning; acquisition and integration of knowledge; refinement and further deepening of knowledge; the significant use of knowledge and productive mental habits.
\end{abstract}

Key words: Ciudad Juárez, Cozumel, Procedural learning, entrepreneurship. businessmen.

*E-mail: noccarempo@gmail.com 


\section{Introducción}

Tocar el tema de las empresas abre un amplio campo para la investigación y el análisis, no solo por el impacto que estas tienen en los distintos sectores de la economía en términos de la valorización de sus productos o servicios, sino también por su incidencia en la generación de empleo, el desarrollo regional, la innovación de productos y procesos y la investigación (Castro, García y Adame, 2015). Es importante analizar tanto el tema de las empresas como a quienes las crean y la manera en que estos individuos se forman-aprenden. Aunado a ello, "hay que admitir que la profesión de empresario es muy compleja, porque ha de atender a un universo de variables muy heterogéneas, generar ciertas estructuras, disponer de capacidad de organización, tomar decisiones, asumir una importante cuota de riesgo y, sobre todo, dirigir hombres, lo que con cierta probabilidad es lo más difícil de todo" (Polaino, 2002, p. 412).

La aportación anterior brinda la pauta para tener en cuenta que ser empresario significa ser capaz de percibir una oportunidad y ante ella formular, libre e independientemente, una decisión de consecución y asignación de recursos naturales, financieros, tecnológicos y humanos necesarios para poner en marcha la empresa, que además de crear valor incremental para la economía, genera trabajo para él y, muchas veces, para otros (Varela, 2001). En ese sentido, Roig y Veciana (2004), además de identificar al empresario como la persona que ha constituido una empresa que funciona y que sobrevive durante años, concuerdan y resaltan la utilidad empírica de la figura de este, como un componente clave del funcionamiento de la economía y del desarrollo económico.

Cabe señalar que, en México, la globalización y la liberalización comercial implican un enorme reto competitivo para las organizaciones, sobre todo para las micro y pequeñas empresas, las cuales exhiben diversas desventajas al hacer frente a este contexto (Pérez, Torralba, Cruz y Martínez, 2016). Dado que este tipo de unidades productivas representan $95.9 \%$ del total de las empresas en el nivel nacional y originan $43 \%$ del empleo en la economía mexicana (INEGI, 2014), el hecho de verse envueltas en un entorno de competencia "las obliga a generar nuevas aptitudes y formas para su organización y funcionamiento, aunque lamentablemente no todos los empresarios entienden esta situación y aplican de manera empírica la gestión de su empresa” (Pérez et al., 2016, p. 20). 
Esto a su vez les exige a los empresarios la capacidad para adaptarse a situaciones diversas; tomar decisiones con consecuencias significativas para ellos mismos, sus empleados y hasta la localidad en la que se ubican; una mayor iniciativa y, finalmente, el incremento de su aprendizaje a través de las experiencias que se presentan dentro de sus actividades diarias.

De lo anterior surge la necesidad de investigar al aprendizaje de los empresarios mexicanos, desde el enfoque del aprendizaje procedimental, ya que, hasta el momento, no han sido detectados estudios similares en el ámbito nacional. Se optó por el enfoque del aprendizaje procedimental porque este tipo aprendizaje está inmerso en los individuos al interactuar con el mundo social y material, en donde las acciones de estos dependen del contexto específico en el cual se desarrollan (Bosch, 2002); adicionalmente, Bosch (2002, p. 36) apunta que "una parte importante del proceso del aprendizaje procedimental es desarrollar la habilidad cognitiva de aprender qué acciones son apropiadas para las diversas situaciones".

La necesidad de realizar esta investigación aumenta al considerar que una de las consecuencias más perjudiciales en la economía nacional es la mortandad de las empresas (Navarrete y Sansores, 2010), de la cual uno de los factores clave puede ser la falta de preparación de los empresarios, lo que impide que estos logren cumplir con los objetivos y metas de sus organizaciones. Un aspecto que se debe tener en cuenta ante esta situación es que por cada 100 empresas que ingresan a la actividad económica anualmente en la república mexicana, solamente 36 sobreviven un año después; al cumplir cinco años, son 28 aquellas que continúan con sus actividades y, luego de 15 años, el total de las empresas que sobreviven se reduce a 15 (INEGI, 2016).

Debido a la problemática planteada, la presente investigación tiene como objetivo efectuar un análisis del aprendizaje procedimental de 12 empresarios mexicanos, de los cuales seis radican en Ciudad Juárez, Chihuahua, y los seis restantes en Cozumel, Quintana Roo. Para recabar información se realizaron entrevistas semiestructuradas, en donde se empleó como guía un instrumento elaborado con base en Marzano (1992). Para el análisis de la información se utilizó la estrategia de estudio de caso múltiple. Finalmente, el estudio muestra cinco dimensiones de aprendizaje procedimental enfocadas a la dinámica empresarial, las cuales pueden contribuir a incrementar la formación de empresarios. 


\section{Antecedentes}

Esta investigación manifiesta una preocupación por el aprendizaje del empresario en el nivel individual. Al analizar la evidencia científica sobre la temática que se trata, se pudo apreciar con claridad la falta de estudios enfocados a la formación de empresarios. Por ende, las limitaciones que se presentan respecto a este tipo de trabajos muestran la necesidad de investigaciones que aborden aquellas experiencias de empresarios que puedan ayudar a formar a los futuros dueños de empresas; ese aprendizaje adquirido a través de experiencias dentro de su organización es el denominado procedimental.

El aprendizaje procedimental se refiere al tipo de aprendizaje que permite adquirir o desarrollar habilidades mediante la ejecución y repetición de una tarea de manera no consciente o no intencional (Gil, Chamorro-Sánchez, DíazMarta y Arroyo-Anlló, 2013). Asimismo, Martínez (2013) lo define como un conjunto de acciones y/o estrategias conscientes, que están planeadas para promover el proceso de enseñanza y aprendizaje, en donde el docente debe facilitar a los participantes el desarrollo secuencial de habilidades para aprender.

De manera semejante, Anderson (2000) afirma que el aprendizaje procedimental puede considerarse como el desarrollo de un sistema de ejecución que no está representado en calidad de información explícita sobre el mundo y que se activa de modo automático ante las demandas de una tarea, como una secuencia de pautas de actuación. De acuerdo con lo anterior, se debe tener en cuenta que este tipo aprendizaje se encuentra inmerso en los individuos al interactuar con el mundo social y material, en donde las acciones de estos dependen del contexto específico donde se desarrollan (Bosch, 2002), por lo tanto es un aprendizaje lento, progresivo e inflexible adquirido mediante la ejecución y repetición de una tarea, que se traduce en una mejora de los resultados o en la facilitación del acceso a cierta información -saber cómo- (Gil et al., 2013).

En relación con la experiencia que adquiere un individuo, Martínez (2013) señala que los procesos de aprendizaje procedimental que una persona puede experimentar en su entorno laboral se presentan a través de experiencias. Hay que mencionar además que el objetivo del aprendizaje procedimental se centra en la "mejora general del procedimiento mental adquirido durante la repetición de la tarea, más que una mejora en el tratamiento de un estímulo presentado con anterioridad” (Gil et al., 2013, p. 404). 
Es por todo ello que se utiliza el enfoque del aprendizaje procedimental para el análisis de las experiencias de los empresarios entrevistados, ya que más que la repetición de acciones para realizar una tarea, este tipo de aprendizaje "debe ser concebido como las distintas formas de pensar para resolver problemas que satisfagan las necesidades del colectivo y a partir de ahí satisfacer las individualidades fomentando la capacidad de enfrentarse a una nueva tarea con una actitud crítica y analítica de los hechos presentes en el contexto" (Martínez, 2013, p. 21).

Para el abordaje de este apartado se empleó la propuesta de Marzano (1992), quien supone que el aprendizaje es producto de la interacción de cinco tipos de pensamiento que denomina "dimensiones del aprendizaje", las cuales son metáforas que se utilizan para expresar cómo trabaja la mente mientras aprende y se enlistan a continuación:

1. Actitudes y percepciones positivas sobre el aprendizaje

2. Adquisición e integración del conocimiento

3. Refinamiento y profundización del conocimiento

4. Aplicación significativa del conocimiento

5. Hábitos mentales productivos

Cabe destacar que no es que ocurran cinco tipos de pensamiento independientes durante el aprendizaje, sino que este es producto de un complejo proceso interactivo, el cual le permite al educador lograr resultados específicos y satisfactorios, ya que pueden preparar a las personas hacia nuevas formas de ver las cosas (Marzano, Pickering, Arredondo, Blackburn, Brandt, Moffett, Paynter, Pollock, y Whisler, 2005). Tales dimensiones se explican en el cuadro 1. 


\section{Cuadro 1.Dimensiones del Aprendizaje Procedimental}

\section{Dimensión}

Actitudes y percepciones positivas sobre el aprendizaje

Adquisición e integración del conocimiento

Refinamiento y profundización del conocimiento

Aplicación significativa del conocimiento

Hábitos mentales productivos
¿En qué consiste?

Experimentar el impacto de actitudes y percepciones en relación con el maestro, sus propias habilidades y el valor de tareas asignadas.

Observar el ejemplo inicial de una acción y llevar a cabo un proceso o demostrar una habilidad, es decir, repetir esa acción.

Examinar y analizar el conocimiento y la información de una forma que permita hacer nuevas conexiones, descubrir o redescubrir sentidos, entender a profundidad nuevos aspectos y aclarar malentendidos; si se presentan dudas, se deben compartir.

El propósito de adquirir conocimiento es ser capaz de darle un uso que tenga sentido. En pocas palabras, cuando se usa el conocimiento para resolver cuestiones específicas que le atañen al individuo, es cuando éste se sumerge en el aprendizaje.

Hacer un análisis con apoyo en las dimensiones del aprendizaje procedimental, puede encajar en tres categorías generales: pensamiento crítico, pensamiento creativo y pensamiento autorregulado.

Si se tiene hábitos mentales que ejemplifiquen el pensamiento crítico, se tiende a ser preciso y buscar precisión, ser claro y buscar claridad y mantener la mente abierta.

Si se tiene hábitos mentales que ejemplifiquen el pensamiento creativo, se tiende a: Perseverar, extender los límites del conocimiento y las capacidades.

De igual manera, si se tiene hábitos mentales que ejemplifiquen el pensamiento autorregulado, se tiende a: Supervisar el propio pensamiento, planear de manera apropiada, identificar y usar los recursos necesarios.

Fuente: Elaboración propia con base en Marzano (1992) y Marzano et al. (2005). 
Con referencia a lo antes expuesto y a efecto de entender la relación entre el análisis del aprendizaje procedimental y las experiencias de los empresarios que se entrevistaron, se menciona la teoría del desarrollo cognoscitivo, la cual fundamenta esta investigación y establece que el conocimiento no es absorbido pasivamente del ambiente y tampoco es procesado en la mente del ser humano ni brota cuando madura, sino que es constituido por el mismo a través de su interacción con sus estructuras mentales y el medio ambiente, más concretamente (Piaget, 1992). Asimismo, desde el punto de vista de Martínez (2013), en esta teoría se involucran los procesos de asimilación y acomodación, representando así aquellas transformaciones de los propios conocimientos; esto ocurre en función de los cambios del medio y de los nuevos datos de la experiencia adquirida, los cuales se incorporan en las estructuras mentales adaptándose y modificando los conocimientos que ya se poseen a los nuevos aspectos de la realidad.

\section{Metodología}

Para efectuar esta investigación se planteó un estudio cualitativo, cuyo objetivo, de acuerdo con Hernández, Fernández y Baptista (2010), es examinar un tema poco investigado o que no ha sido abordado con anterioridad. El estudio se caracteriza por ser descriptivo y exploratorio; respecto a su diseño, se debe agregar que es no experimental, puesto que se realizó sin manipular deliberadamente variables y, referente al tiempo, es transversal, debido a que se recolectó información en un determinado momento.

Se utilizó la estrategia de investigación denominada estudio de caso múltiple, cuyo interés, explica Stake (1998), se centra en indagar un fenómeno, población o condición general a partir del estudio intensivo de varios casos a la vez. Además, el estudio de casos es una investigación empírica que aborda un fenómeno contemporáneo dentro de su contexto de la vida real, en especial cuando los límites entre el fenómeno y su contexto no son claramente evidentes (Yin, 1994).

Para recabar información se realizaron entrevistas semiestructuradas durante mayo, junio y julio de 2014, donde se empleó como guía un instrumento elaborado con base en Marzano (1992), el cual comprende de tres apartados: datos personales del empresario, aspectos de identidad del empresario y aprendizaje procedimental. 
Respecto al análisis de la información obtenida, se utilizó el software Atlas.ti, con el cual se llevó a cabo un proceso de codificación de la información, en donde se detectaron códigos in vivo y teóricos, respectivamente, obtenidos a partir de las entrevistas semiestructuradas realizadas y los fragmentos arquetípicos extraídos de ellas; de ahí se elaboraron cuadros analíticos sintéticos.

La población muestral consta de 12 empresarios ubicados en las zonas norte y sur de la república mexicana (seis radican en Cozumel, Quintana Roo, y seis en Ciudad Juárez, Chihuahua). Debido a la situación de inseguridad que se presentaba en Ciudad Juárez en ese periodo, el muestreo fue dirigido. De acuerdo con Patton (1988), este muestreo tiene su origen en consideraciones de tipo práctico en las cuales se busca obtener la mejor información en el menor tiempo posible, según las circunstancias concretas que rodean tanto al investigador como a los sujetos o grupos investigados. La mayoría de los empresarios entrevistados pertenece al ramo de la industria restaurantera, habiendo también aquellos que son dueños de una tienda de abarrotes, una pastelería, una joyería, un centro cambiario, una ferretería, una purificadora, una empresa de servicios de remodelación, y un par de franquicias (una dedicada a la venta de pintura y la otra a la preparación y venta de comida rápida).

\section{Resultados}

Con base en los resultados obtenidos al realizarse las entrevistas semiestructuradas y la codificación de estas, se presentan a continuación los aspectos más destacados de los apartados datos generales del empresario y aspectos de su identidad.

\section{Datos generales del empresario}

Dentro de este apartado se identifica la procedencia de los empresarios, quienes son originarios de distintas partes de la república mexicana, como Ciudad Juárez, Chihuahua, Torreón, Mérida, Cozumel, Cancún y Playa del Carmen. Un hallazgo importante es que, de un total de 12 empresarios entrevistados, cuatro son mujeres y ocho varones; respecto a su edad, el rango comprende de los 27 a los 59 años de edad. En relación con el tiempo de existencia de su negocio, se encontró que la empresa más joven tenía seis años de haberse formado, y 
aquella con la máxima cantidad de años tenía 41; algunos de los entrevistados mencionaron que sus padres también se dedicaban a la misma actividad económica.

\section{Aspectos de identidad del empresario}

Se detectó en los empresarios una enorme inclinación hacia el deseo y la necesidad de tener un negocio propio, independientemente del giro al que pertenezca y la ubicación de la empresa, sustentándose en la cuestión de que desde la niñez se tenía el sueño de establecer un negocio, así como también el apoyo y ánimo de familiares del entrevistado en formar una empresa propia. De acuerdo con Rodríguez y Santos (2008), la motivación para emprender, considerada como el impulso interior que empuja a los individuos a crear una empresa, varía dependiendo de las características personales del empresario y de las circunstancias de su entorno.

Otro de los factores que ayudaron a que los entrevistados lograran crear una empresa es que pasaron gran parte, si no es que toda su vida, en el negocio que su familia tenía; en este caso, Lloret y Moya (2013) afirman que esta situación suele resultar muy beneficiosa para las empresas familiares, ya que la propiedad se hereda, mientras que el talento para la gestión no. En contraste, otros empresarios mencionan que estuvieron a cargo del negocio durante periodos considerables, en donde aprendieron a manejarlo y de ahí surgió la idea de abrir su propio establecimiento, continuando con la tradición familiar.

De los 12 entrevistados, cinco tuvieron padres empresarios que fungieron como facilitadores, o bien, guías de formación para su aprendizaje, aspecto que será abordado con mayor profundidad dentro del apartado de discusión, en la dimensión 2. Al respecto, Quintana (2001) sostiene que uno de los aspectos más valorados por los empresarios en la creación de empresa es la red de apoyo moral que reciben de la familia y amigos; esa red suele provenir de tres de sus ámbitos más cercanos: el padre, el cónyuge y los hijos. Por lo tanto, al tener la familia un impacto en el éxito de la empresa (creación y consolidación), también esta deberá contemplar sus necesidades y exigencias para afrontar las relaciones que se establecen entre ambas. 


\section{Discusión}

Para discutir la información obtenida respecto al aprendizaje procedimental, se dividirá según las dimensiones de este mencionadas dentro del marco teórico (véase cuadro 1), las cuales se presentan a continuación.

\section{Dimensión 1: Actitudes y percepciones positivas sobre el aprendizaje}

En esta dimensión se consideraron los códigos teóricos "Actitud” y "Ambiente para adquirir nuevos conocimientos". Con base en ellos se determina que para que el empresario pueda adquirir nuevos conocimientos, debe adoptar una actitud positiva y estar con la mente abierta. Además, su formación se debe centrar en la actualización de sus conocimientos; la mayoría de los encuestados en el área fronteriza establece que el empresario debe estar actualizado, para enfrentar los cambios culturales, políticos y económicos de su entorno y de esta manera no quedarse rezagado dentro del mercado.

Este hallazgo coincide con Elosúa (1993), dado que menciona algunas actitudes que deben adoptarse para el proceso de enseñanza en un aula de clase: actitud tolerante, respetuosa y positiva hacia la novedad e interés por ampliar las experiencias; actitud abierta al cuestionamiento de las cosas y un sentido vivo para preguntarse por todo.

Cabe señalar que los entrevistados respondieron que un ambiente óptimo donde se pueden adquirir nuevos conocimientos debe ser como el que posee un aula de clase, donde debe predominar el orden, el respeto y la confianza para retroalimentar al alumno. Esto coincide con De Giraldo y Mera (2000), quienes apuntan que un ambiente escolar agradable, amplio, ordenado, comprensivo y estimulante le facilita al estudiante satisfacer ciertas necesidades básicas relacionadas con habilidades y responsabilidades; también favorece la motivación y la socialización.

\section{Dimensión 2: Adquisición e integración del conocimiento}

Para esta dimensión se tuvieron en cuenta los códigos teóricos "Muestra previa antes de establecer el negocio", "Actividad donde estableció pasos para llevarse a cabo" y "Problemas que contribuyeron en aprendizaje". Acorde con Marzano et al. (2005), sin un modelo inicial, aprender una habilidad o un proceso puede 
ser caótico y absorbente, porque en esencia se trata de un proceso de prueba y error; es importante contar con la presencia y el apoyo de un guía o facilitador, el cual debe estar consciente de, y con sensibilidad hacia, el hecho de que un proceso o habilidad que está enseñando es nuevo para el aprendiz.

Los referentes encontrados acerca del rol de facilitadores o guías de proceso de los cuales los empresarios aprendieron son los siguientes:

- Abuelo: el negocio inicialmente fue fundado por el abuelo, el cual heredó al padre o la madre de los entrevistados. Se detectó que este miembro de la familia era, en su mayoría, el propietario de las tierras en donde se situaba el establecimiento comercial; su función principal fue la creación del producto en particular, adquirir cierto reconocimiento en el producto y/o servicio brindado y, finalmente, permitir que el o la sucesora continuara con el negocio, así como autorizar la implementación de algunas mejoras respecto a los procedimientos utilizados al comienzo de la empresa.

- Padres: estos facilitadores sobresalieron en la mayoría de los resultados obtenidos. En el caso de la madre del empresario entrevistado, su función era formar a sus hijos en el trato al cliente, donde debe prevalecer la amabilidad y paciencia hacia el mismo; recomendar y, hasta cierto punto, presionar a sus hijos para estar al pendiente de las empresas que se dedicaran a la misma actividad, y respetar los procedimientos ya establecidos en caso de elaborar algún producto. Hay que mencionar también que se observó la importancia que le brindaba la figura materna a la obtención de permisos necesarios por parte del gobierno para que el negocio pudiera funcionar acorde a la ley. En lo que corresponde al padre, se encontró que en un principio desempeñó el rol de socio con el creador de la empresa, una vez que ya dominaba ciertas actividades llevadas a cabo en la organización; se centra primordialmente en mejorar e innovar los procedimientos de elaboración del producto y la atención al cliente. El entrevistado acompañaba a su padre desde la niñez; con el paso de los años y la ayuda de su progenitor conoció y entendió el funcionamiento del negocio, en razón de una relación más estrecha en comparación con la madre; los aspectos que resaltan según la información obtenida son 
manejo de mercancía, pago de impuestos, problemas comunes y aspectos de seguridad. De ahí se procede a dejar al mando de la entidad al entrevistado y emergen como consultores particulares de sus hijos debido a su experiencia en el negocio.

- Antiguos jefes-patrones: en el caso de los jefes o patrones de empleos obtenidos anteriormente a la creación de su empresa, se debe subrayar que el conocimiento y dominio de una actividad o la elaboración de algún producto fue adquirido con ayuda de ellos. El paso de los años en una empresa en particular contribuyó a que el empresario pudiera ejercer el dominio de ciertas actividades, en las que destacan manejo de personal, procesos de elaboración de productos-recetas, higiene, compras y reportes de ventas. Por consiguiente, el empresario hace mención del momento en el cual decide emprender acorde a su experiencia que funge como un soporte para el manejo del negocio de reciente creación; esta situación se caracteriza por ser un tanto más especializada en comparación con las anteriores.

- Franquicia: al hacer referencia sobre una franquicia, el empresario entrevistado señaló que recibió una preparación en el extranjero, fue entrenado desde lo más básico hasta concluir con el manejo de sistemas y la adopción de un plan de negocios; no contó con el apoyo de un facilitador, sino de varios especialistas pertenecientes al corporativo. También se le proporciona un manual de operaciones, el cual puede apoyarlo en cualquier momento.

De acuerdo con lo anteriormente planteado, se encontró que en más de la mitad de los empresarios existió un patrón previo, que les sirvió como ejemplo de cierta manera a la hora de establecer su negocio. De estos patrones sobresalen cuatro opciones donde el empresario adquiere un conocimiento: influencia familiar, antiguos empleos, patrón de franquicia y por sí mismo.

En el caso de la influencia familiar, el papel de facilitador o guía lo asumen el padre, la madre o bien algún familiar directo del empresario; el individuo ha adquirido experiencia al relacionarse directamente con el negocio que su familia ha manejado, ya que es común que haya pasado largos periodos en él. Venter, Boshoff y Maas (2005) sostienen que una buena relación familiar entre el 
descendiente y sus padres favorece la relación laboral, de lo cual se desprende que esta última apoyaría, a su vez, la transferencia de conocimiento tácito del padre al descendiente.

Otro rasgo a considerar es que el empresario trabajó previamente en una o distintas empresas, donde adquirió experiencia en diferentes áreas, lo que le beneficia a la hora de montar su propio negocio, ya que aplica conocimientos que logró dominar en el pasado. Lozano (2008) afirma que la experiencia laboral externa contribuye a ganar autoconfianza, lo cual va forjando un comportamiento más autónomo por parte del empresario, sin olvidar que favorece su formación de líder.

Un aspecto que difiere un poco de los casos anteriores es la adquisición de una franquicia; los empresarios entrevistados comentaron que la marca es un factor clave en las ventas, lo cual otorga ciertos beneficios dado que el corporativo ya brinda un plan de negocios efectivo; el aspecto negativo de esta situación es que en ocasiones la visión del empresario se puede observar un tanto limitada por el corporativo que se encarga de llevar a cabo evaluaciones a las sucursales de su marca, ya que en caso de no cumplir con los requerimientos necesarios por ser marca registrada, se puede ser amonestado e incluso llegar a la suspensión de licencia.

Mariz y García (2003) argumentan que un franquiciado constituye, en muchos aspectos, un ejemplo de empresario independiente; el contrato que lo une a la cadena -normalmente de larga duración- le permite explotar una marca, obtener asistencia empresarial del corporativo y apropiarse de los beneficios generados por su unidad.

Otro punto importante de abordar cuando el empresario aprende por sí mismo es el que algunos entrevistados mencionaron: nadie les enseñó lo que era tener una empresa; ante ello plantean que todo ha sido decidido de la manera en que se consideró mejor, lo cual es más complicado, ya que no cuentan con una guía o alguna experiencia previa en la dinámica empresarial. También aclaran que la educación siempre será considerada como un soporte al momento de crear un negocio.

Por lo tanto, Durand (2008) argumenta que en las dos últimas décadas se ha visto triunfar a personas que, saliendo casi de la nada, se han convertido en mitos empresariales. Es aquí donde se presenta la viabilidad del proyecto de la 
empresa, que "radica en el equilibrio entre los cinco elementos base: la idea, la planificación, los recursos, la gestión y, en el centro, el empresario” (Durand, 2008, p. 4).

El siguiente aspecto trata de aquellos problemas o situaciones que le permitieron aprender al grupo de entrevistados. Respecto a Cozumel, en la temporada baja, como su nombre lo indica, las ventas disminuyen considerablemente; este periodo trae consigo la inseguridad y el desempleo. Arroyo y Gutiérrez (2007) reafirman esta situación al señalar que la temporada baja es la causa de la caída de las ventas y el despido de empleados en el sector hotelero, principal fuente de empleo en Cozumel.

En Ciudad Juárez, la inseguridad es un problema que afectó completamente la vida empresarial de la ciudad. Márquez, Cuevas y Vilchis (2013) explican que el cierre de los negocios derivado de la inseguridad y la extorsión ha provocado la pérdida de empleos o el desplazamiento de los trabajadores a otros sectores; dentro del sector terciario los cambios más significativos fueron un crecimiento del comercio hasta de $30 \%$ en 2005, y, para 2009, una caída hasta de $24 \%$; particularmente los comerciantes de Ciudad Juárez han cerrado múltiples negocios. Otros problemas de menor grado fueron la falta de capacitación y la rotación del personal.

\section{Dimensión 3: Refinamiento y profundización del conocimiento}

Dentro de la dimensión 3 se contemplaron los códigos teóricos "Deducir problemas” y "Análisis de errores". Al preguntarle a los empresarios de la región cozumeleña cómo deducían que la temporada baja se aproximaba, respondieron que por un periodo de meses: entre septiembre, octubre y noviembre bajan las ventas, ya que es cuando dejan de llegar cruceros, y se comienza a notar un sobrante considerable de mercancía en los diversos restaurantes, lo cual se liga directamente con la afirmación de Palafox y Segrado (2008), quienes sostienen que la temporada baja comprende los meses de septiembre a noviembre. A la par, deducir el problema de la inseguridad en Ciudad Juárez resulta menos complicado ya que es común apreciar el aumento de asaltos alrededor de la empresa, generando una desconfianza permanente hacia los demás, hasta llegar al punto de solicitar al empresario pagar su "cuota", o bien, cerrar puertas.

En este sentido, "dado que el $32.8 \%$ de los negocios en Ciudad Juárez son micronegocios, este tipo tan vulnerable de negocio es el que se ve, incluso 
hasta hoy en día, afectado por la situación de inseguridad en Juárez; son estos pequeños negocios los que tienen que cerrar puertas por ser incapaces de cubrir los pagos conocidos como 'cuotas' que cobran los delincuentes por uso de piso, desapareciendo fuentes de empleo” (Márquez, Cuevas y Vilchis, 2013, p. 223).

Respecto a la falta de capacitación de los empleados, con base en los empresarios entrevistados, se deduce cuando el trabajador no muestra un dominio del conocimiento en cuanto al servicio, producto o actividad. Paralelamente a este hallazgo, Méndez (2002) complementa el análisis de esta problemática al indicar que es muy importante preguntarse si el empleado tiene la habilidad y el conocimiento para realizar sus funciones, ya que pueden existir problemas en su desempeño.

Con relación a la rotación del personal, los empresarios de Ciudad Juárez compartieron que su empresa se ve afectada por esta situación debido principalmente al regreso a clases, y por las vacaciones navideñas. En contraste, en Cozumel la rotación de personal se da sobre todo por la búsqueda de un mejor empleo, el hecho de dejar la isla, y la propia condición de vivir por temporadas alta y baja. Esto coincide con los resultados de Arroyo y Gutiérrez (2007, p. 73), quienes señalan que esta problemática es ocasionada "según los responsables de recursos humanos, por la búsqueda de mejores empleos, migración y la temporalidad”.

\section{Dimensión 4: Aplicación significativa del conocimiento}

En esta dimensión se observaron los códigos teóricos “Toma de decisiones” y “Capacitación”. En cuanto a Cozumel, a la hora de tomar decisiones, la mayoría de los empresarios dijo que se comenta primero con la familia, después acuden con algún amigo que tenga experiencia en el caso y se le pide consejo.

Otras acciones que se llevan a cabo es el chequeo del sistema de control interno, acudir a una consulta con el contador y entre ambos decidir o bien apegarse a las políticas que el patrón familiar que siguen ha manejado desde sus inicios. De igual manera, la consulta familiar se hace presente en los empresarios de Ciudad Juárez a la hora de tomar decisiones; aunque con menor frecuencia, también preguntan a los padres o, en su caso, a la pareja. Normalmente con quien se asesoran suele ser el fundador o parte de los fundadores de la empresa, ya que se debe cumplir con determinados códigos familiares y a su vez respetar 
el entorno familiar con relación a sus necesidades y exigencias sin perder de vista a la empresa como tal (Basco, 2006). Asimismo se encontró que hay un apego del reglamento interno de algunas empresas a la Ley Federal del Trabajo, utilizándolo como guía a la hora de tomar decisiones, esto es, cuando existe una constante rotación de personal.

Tanto en Cozumel como en Ciudad Juárez se destacan las mismas formas de capacitación a empleados: se le da prioridad a un entrenamiento individual, para lo cual se explica previamente a la persona la actividad a realizar, por un lapso de dos semanas, y se le pone a prueba un mes antes de ofrecerle el contrato, en ese mes se le hacen preguntas y se le retroalimenta en caso de necesitarlo.

\section{Dimensión 5: Hábitos mentales productivos}

Finalmente, para la dimensión 5 se atendieron los códigos teóricos "Impulsos", "Nuevas maneras de ver una situación” y "Evaluar acciones”. Sobre el hábito mental que un empresario puede experimentar, en este caso el impulso, en ambas localidades se mencionó que no era correcto, ya que puede provocar que el empresario cometa errores que dañen fuertemente al negocio. Asimismo, los entrevistados comentaron que se deben generar nuevas maneras de ver una situación en donde se contemple todo lo que rodea a la situación para darle la solución más adecuada posible, y si no están seguros, pedir asesoría, pues siempre existirá algún especialista que podrá ayudar. Por otro lado, como empresarios, se deben dar a la tarea de revisar periódicamente qué tanto ha crecido su negocio y que tanto se ha logrado como empresario, y así evaluar sus acciones.

\section{Conclusión}

Con base en este análisis de aprendizaje procedimental se concluye que para que el empresario pueda adquirir nuevos conocimientos, debe adoptar una actitud positiva, buena y estar con la mente abierta. Se consideró que un ambiente óptimo para ello es aquel similar al del aula de clase, en el cual debe haber orden, respeto y confianza. Se destacan cuatro opciones donde el empresario recibe una instrucción: influencia familiar, antiguos empleos, patrón de franquicia y por sí mismos. La influencia familiar se presenta cuando el empresario ha adquirido 
experiencia al relacionarse directamente con el negocio que su familia ha manejado; es común que el individuo haya pasado largos periodos en esa empresa; los facilitadores o guías en este caso son el abuelo y los padres. Por otro lado, al trabajar el empresario en una o distintas organizaciones, obtiene experiencia en diferentes áreas, lo cual le beneficia a la hora de montar su propio negocio, ya que aplicará conocimientos que logró dominar en el pasado; sus facilitadores fueron los antiguos jefes-patrones.

A su vez, el empresario que adquiere una franquicia debe apegarse a una serie de reglas que el corporativo establece, ya que cada cierto tiempo es evaluado para conservar la licencia de la marca; el aporte de los facilitadores que pertenecen al corporativo se ve reflejado en un manual de operaciones que se le proporcionó. Cuando el empresario aprende por sí mismo, se alude a la educación y a la viabilidad del proyecto. La primera es considerada como un soporte al momento de crear una empresa, y en cuanto a la segunda, esta radica en el equilibrio entre los cinco elementos base: la idea, la planificación, los recursos, la gestión y, en el centro, el empresario. Por otra parte, de los problemas que permitieron aprender al grupo de empresarios entrevistados sobresalen la temporada baja en Cozumel y la inseguridad en Ciudad Juárez.

Es evidente que, para tomar una decisión, los empresarios consultan con su familia, apoyo profesional y/o empleado de confianza, teniendo en cuenta las opciones más convenientes para el bienestar de su empresa. En atención a los procesos de capacitación, el empresario le da prioridad a un entrenamiento individual para sus empleados, realizando previamente una junta con ellos donde se les explica la actividad, y después brindándoles atención personalizada. De los hábitos mentales productivos se enfatiza que el empresario no debe actuar de manera impulsiva, ya que puede cometer errores que dañen fuertemente a la empresa; otra de las acciones a considerar es que debe contemplar todo lo que rodea a la situación y darle una solución, la más adecuada posible, y en caso de no estar seguro puede pedir asesoría, ya que siempre existirá algún especialista que lo ayude. Hay que señalar además que el empresario debe preocuparse por evaluar sus acciones, debe saber qué piensan sus clientes y empleados y verificar si las necesidades de los clientes se satisfacen.

Acorde con el anexo, el cual contiene las dimensiones del aprendizaje procedimental en empresarios, que se construyeron con base en los resultados 
obtenidos a través de entrevistas semiestructuradas, se estima, a diferencia de Marzano et al. (2005), que la segunda dimensión es la más importante para el aprendizaje procedimental de un empresario; esto se debe a que en este apartado el empresario recibirá nuevos conocimientos, aprenderá a apropiarse de las nuevas ideas con ayuda de sus facilitadores, se le presentarán algunos problemas para lograr el entendimiento completo de las diferentes situaciones y finalmente obtendrá, en su mayoría, el dominio de las situaciones y de los problemas que afrontó. Lo antes planteado se vincula directamente con la teoría del desarrollo cognitivo de Piaget (1992), ya que el empresario incorpora una experiencia como información nueva al interior de sus estructuras cognoscitivas, ajustando el conocimiento para un mejor desarrollo en sus acciones, mecanismos conocidos como asimilación y acomodación.

Por último, teniendo en cuenta que las dimensiones del aprendizaje procedimental en empresarios se construyeron a partir de un estudio meramente cualitativo, estas se generalizan hacia la teoría no hacia la población, por lo tanto existen limitaciones respecto al aprendizaje procedimental de otros empresarios que residan en localidades distintas a las aquí tratadas.

\section{Fuentes consultadas}

Anderson, J. (2000). Learning and Memory: An Integrated Approach. Nueva York: Wiley.

Arroyo, L. y Gutiérrez, E. (2007). El empleo en el sector hotelero de Cozumel y su impacto en la comunidad. Teoría y Praxis, 3, 65-76.

Basco, J. (2006). La investigación en la empresa familiar: "un debate sobre la existencia de un campo independiente”. Investigaciones Europeas de Dirección y Economía de la Empresa, 12, 33-54.

Bosch, M. (2002). La gestión del conocimiento en el medio digital: viejos problemas de tratamiento de información y aspectos nuevos. Ciencias de la Información, 3(1), 35-43.

Castro, M., García, M. y Adame, M. (2015). Hacia una comprensión de los conceptos de emprendedores y empresarios. Suma de Negocios, 6(13), 98-107.

Durand, T. (2008). El perfil y las habilidades del empresario. Madrid: Departamento de Publicaciones del IE Business School. 
Elosúa, M. (1993). Estrategias para enseñar y aprender a pensar. Madrid: Narcea. Gil, R., Chamorro-Sánchez, J., Díaz-Marta, J. y Arroyo-Anlló, E. (2013). Memoria procedimental en pacientes con enfermedad de Alzheimer. Revista Médica del Instituto Mexicano del Seguro Social, 51, 403-413.

Giraldo, L. de y Mera, R. (2000). Clima social escolar: percepción del estudiante. Revista Salud y Educación, 31, 23-27.

Hernández, R., Fernández, C. y Baptista, P. (2010). Metodología de la investigación (5a ed.). México: McGraw-Hill.

INEGI. (2014). Censos económicos 2014. Micro, pequeña, mediana y gran empresa: estratificación de los establecimientos. México: Instituto Nacional de Estadística y Geografía.

INEGI. (2016). Investigación: Esperanza de vida de los negocios en México. Instituto Nacional de Estadística y Geografía. Recuperado de http://www.inegi.org.mx/inegi/contenidos/investigacion/Experimentales/Esperanza/ default.aspx [2016, 25 de julio].

Lloret, J. y Moya, M. (2013). Empresa familiar: cinco claves para perdurar con éxito. Revista de Negocios del IEEM, 16(5), 18-20.

Lozano, M. (2008). Elementos del proceso de formación de descendientes antes de su vinculación a la empresa familiar: un estudio de casos colombianos. Cuadernos de Administración, 21(37), 243-268.

Mariz, R. y García, M. (2003). Una aproximación contractual al estudio de las cadenas de franquicia. En Anales de economía aplicada (pp. 158-181). Asociación Española de Economía Aplicada.

Márquez, B., Cuevas, T. y Vilchis, A. (2013). Turismo Fronterizo. Migración de negocios de entretenimiento y restaurantes de Ciudad Juárez, México-El Paso E.U.A. como estrategia de competitividad. En K. Amador, L. Arrollo y R. Segrado (coord.), Educación, investigación y experiencia para la competitividad turística. (1), 219-230.

Martínez, H. (2013). Construcción del aprendizaje procedimental en estudiantes de las escuelas técnicas industriales robinsonianas (Tesis de maestría). Universidad del Zulia, Maracaibo.

Marzano, R. (1992). A Different Kind of Classroom: Teaching with Dimensions of Learning. Alexandria: AscD.

Marzano, R., Pickering, D., Arredondo, D., Blackburn, G., Brandt, R., Moffett, C., Paynter, D., Pollock, J. y Whisler, J.(2005). Dimensiones del aprendizaje. 
Guadalajara, México: Instituto Tecnológico y de Estudios Superiores de Occidente.

Méndez, E. (2002). Importancia de la detección de necesidades de capacitación y formación de la ccss. Revista de Ciencias Administrativas y Financieras de la Seguridad Social, 10(2), 65-72.

Navarrete, E. y Sansores, E. (2010). El fracaso de las micro, pequeñas y medianas empresas en Quintana Roo, México: un análisis multivariante. Revista Internacional Administración \& Finanzas, 4(3), 21-33.

Palafox, A. y Segrado, R. (2008). Capacidad de carga turística: Alternativa para el desarrollo sustentable de Cozumel. Revista Turismo \& Desenvolvimento, 10, 137-148.

Patton, M. (1988). How to Use Qualitative Methods in Evaluation. Newbury Park: Sage.

Pérez, A., Torralba, A., Cruz, J. y Martínez, I. (2016). Las fuentes de financiamiento en las microempresas de puebla, México. The founding sources in SME's of Puebla, Mexico. Tec Empresarial, 10(1), 19-28.

Piaget, J. (1992). El enfoque constructivista de Piaget. Buenos Aires: Colihue.

Polaino, A. (2002). La salud mental del empresario. Revista Empresa y Humanismo, 5(2), 411-446.

Quintana, C. (2001). Dimensiones del éxito de las empresas emprendedoras. Investigaciones Europeas de Dirección y Economía de la Empresa, 7(2), 139-158.

Rodríguez, M. y Santos, F. (2008). La actividad emprendedora de las mujeres y el proceso de creación de empresas. Información Comercial Española. Revista de Economía, 841, 117-132.

Roig, S. y Veciana, J. (2004). The Entrepreneur and Starting Up New R\&D\&I Business. Valencia: Universidad de Valencia.

Stake, R. (1998). Investigación con estudio de casos. Madrid: Ediciones Morata. Varela, R. (2001). Innovación empresarial: arte y ciencia en la creación de empresas. Bogotá: Pearson Educación.

Venter, E., Boshoff, G. y Maas, G. (2005). The influence of successor-related factors on the succession process in small and medium-sized family businesses. Family Business Review, 18(4), 283-303.

Yin, R. (1994). Case Study Research: Design and Methods. Thousand Oaks: Sage. 


\section{Anexo}

\section{Dimensiones del aprendizaje procedimental en empresarios}

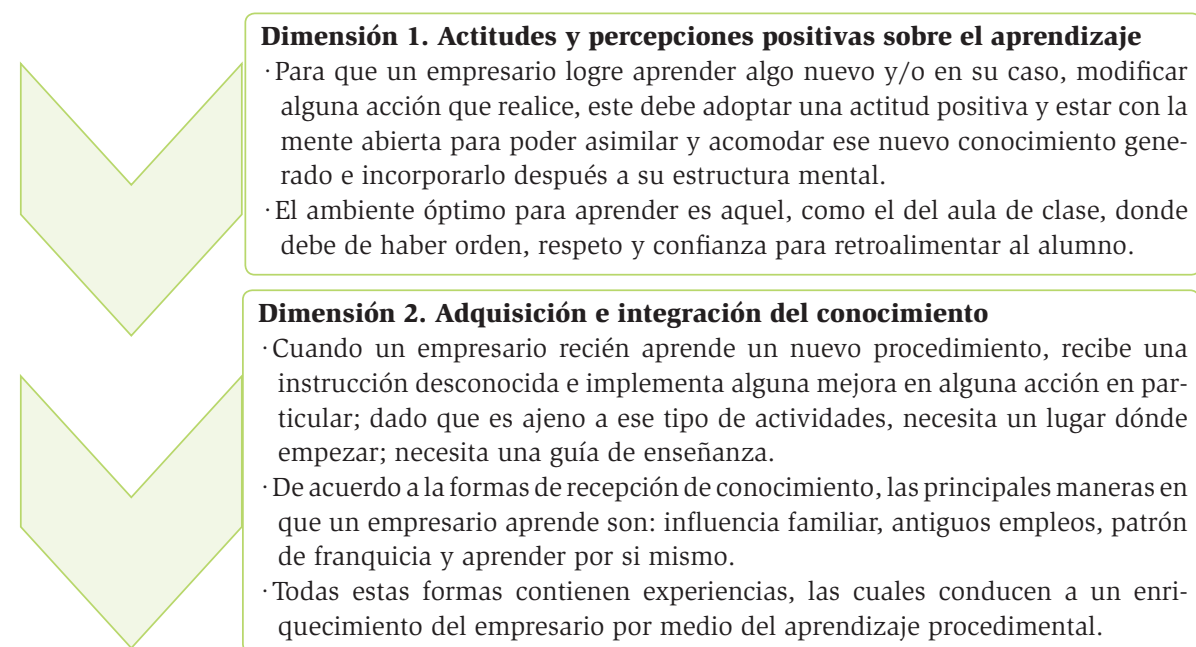

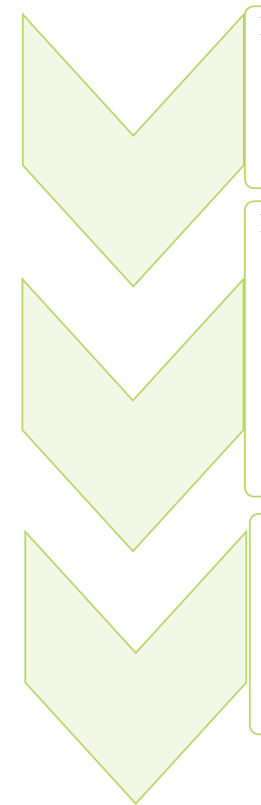

\section{Dimensión 3. Refinamiento y profundización del conocimiento}

- Una vez integrado el conocimiento a la estructura mental, el empresario debe centrarse en deducir y prevenir problemas, así como hacer un análisis periódico de los posibles errores que se pueden presentar dentro de su empresa, todo ello con el fin de evitar esas situaciones.

\section{Dimensión 4: Aplicación significativa del conocimiento}

- El empresario se encargara de generar y aplicar criterios y conocimientos para elegir la mejor opción para su empresa al momento de capacitar a su personal, se hace mención del entrenamiento individual-personalizado a manera de sugerencia.

- En el caso de la toma de decisiones, los empresarios deben consultar con su familia, apoyo profesional y/o empleado de confianza, teniendo en cuenta las opciones más convenientes para el bienestar de su empresa.

\section{Dimensión 5. Hábitos mentales productivos}

- En este punto, el empresario debe identificar aquellos hábitos mentales productivos que, junto con las actitudes y las percepciones, forman un telón de fondo para su proceso de aprendizaje.

Se destacan los hábitos siguientes: no actuar mediante impulsos, adquirir nuevas maneras de ver una situación y evaluar sus acciones constantemente.

Fuente: Elaboración propia con base en entrevistas semiestructuradas.

FIGURA 1: Dimensiones del aprendizaje procedimental en empresarios 